\title{
The Hamburg/ESO R-process Enhanced Star survey (HERES): Project Overview, and New r-II Stars $\dagger$
}

\author{
N. Christlieb ${ }^{1,3}$, T.C. Beers ${ }^{2}$, P.S. Barklem ${ }^{3}$, M.S. Bessell ${ }^{4}$, V. Hill ${ }^{5}$, \\ J. Holmberg ${ }^{6,7}$, A.J. Korn ${ }^{3,8}$, B. Marsteller ${ }^{2}$, L. Mashonkina ${ }^{9,8}$, \\ S. Rossi ${ }^{10}$, F.-J. Zickgraf ${ }^{1}$, K.-L. Kratz ${ }^{11,12}$, B. Nordström ${ }^{6,13}$, \\ B. Pfeiffer ${ }^{11,12}$, J. Rhee ${ }^{14,15}$, and S.G. Ryan ${ }^{16}$ \\ ${ }^{1}$ Hamburger Sternwarte, Universität Hamburg, Germany \\ email: nchristlieb@hs.uni-hamburg.de \\ ${ }^{2}$ Department of Physics and Astronomy and JINA: Joint Institute for Nuclear Astrophysics, \\ Michigan State University, USA \\ ${ }^{3}$ Department of Astronomy and Space Physics, Uppsala University, Sweden \\ ${ }^{4}$ Research School of Astronomy \& Astrophysics, Australian National University, Australia \\ ${ }^{5}$ Observatoire de Paris, GEPI and URA $8111 \mathrm{du}$ CNRS, France \\ ${ }^{6}$ Niels Bohr Institute, Copenhagen University, Denmark \\ ${ }^{7}$ Nordic Optical Telescope Scientific Association, Spain \\ ${ }^{8}$ Universitäts-Sternwarte München, Germany \\ ${ }^{9}$ Institute of Astronomy, Russian Academy of Sciences, Russia \\ ${ }^{10}$ Departamento de Astronomia Instituto de Astronomia, Geofísica e Ciências Atmosféricas, \\ Universidade de São Paulo, Brazil \\ ${ }^{11}$ VISTARS HGF - Virtual Institute for Nuclear Structure and Nuclear Astrophysics, Mainz \\ ${ }^{12}$ Institut für Kernchemie, Universität Mainz, Germany \\ ${ }^{13}$ Lund Observatory, Lund University, Sweden \\ ${ }^{14}$ Center for Space Astrophysics, Yonsei University, Korea \\ ${ }^{15}$ Space Astrophysics Laboratory, California Institute of Technology, USA \\ ${ }^{16}$ Department of Physics and Astronomy, Open University, UK
}

\begin{abstract}
We report on a dedicated effort to identify and study metal-poor stars that are strongly enhanced in r-process elements $([\mathrm{r} / \mathrm{Fe}]>+1.0$ dex; hereafter $\mathrm{r}$-II stars $)$, the Hamburg/ESO R-process Enhanced Star survey (HERES). In a sample of 253 confirmed metal-poor stars for which "snapshot" spectra $(R \sim 20,000 ; S / N \sim 50 / 1$ per pixel) were obtained with VLT/UVES, and abundances were determined in an automated fashion using the methods of Barklem et al. (2005), we identified eight new r-II stars. They are now being studied in detail by means of higher resolution and $S / N$ spectroscopy. The new r-II stars have metallicities in the range $-3.2<[\mathrm{Fe} / \mathrm{H}]<-2.6$. Future searches for $\mathrm{r}$-II stars should therefore focus on stars in this $[\mathrm{Fe} / \mathrm{H}]$ range. Moderately r-process enhanced stars (i.e., $+0.3 \mathrm{dex}<[\mathrm{r} / \mathrm{Fe}]<+1.0 \mathrm{dex}$; r-I stars) were found at metallicities as high as $[\mathrm{Fe} / \mathrm{H}]=-1.5$. The $[\mathrm{Fe} / \mathrm{H}]$ ranges in which $\mathrm{r}-\mathrm{I}$ and $\mathrm{r}$-II stars can be found may provide an important constraint for the identification of the site(s) of the r-process(es).
\end{abstract}

Keywords. stars: abundances - stars: Population II - Galaxy: abundances - Galaxy: evolution Galaxy: halo

$\dagger$ Based in large part on observations collected at the European Southern Observatory, Paranal, Chile (proposals number 68.B-0320 and 170.D-0010). 


\section{Introduction}

The two currently largest wide-angle spectroscopic surveys for metal-poor stars are the HK survey of Beers and collaborators (Beers et al. 1985, 1992), and the Hamburg/ESO Survey (HES; Wisotzki et al. 2000; Christlieb 2003). So far, more than 300 stars confirmed by moderate-resolution spectroscopy to have $[\mathrm{Fe} / \mathrm{H}]<-3.0$ have been identified in these surveys (see Beers et al., this volume). In the HES, the two most iron-deficient stars, $\mathrm{HE} 1327-2326\left([\mathrm{Fe} / \mathrm{H}]_{\mathrm{NLTE}}=-5.4\right.$; Frebel et al. 2005; Aoki et al., in preparation; see also Frebel et al., this volume) and $\mathrm{HE} 0107-5240\left([\mathrm{Fe} / \mathrm{H}]_{\mathrm{NLTE}}=-5.3\right.$; Christlieb et al. 2002, 2004) have been found.

Many astrophysical studies require investigations of sub-classes of metal-poor stars. For example, for nucleochronometric age dating, and/or studies of the r-process, metalpoor stars with moderate $(0.3 \leqslant[\mathrm{Eu} / \mathrm{Fe}] \leqslant+1.0$ and $[\mathrm{Ba} / \mathrm{Eu}]<0$; hereafter $\mathrm{r}-\mathrm{I}$ stars $)$ or strong $([\mathrm{Eu} / \mathrm{Fe}]>+1.0$ and $[\mathrm{Ba} / \mathrm{Eu}]<0$; hereafter r-II stars) enhancements of $\mathrm{r}$ process elements are most suitable. In this contribution we report on a dedicated effort to identify such stars by means of "snapshot" spectroscopy; i.e., spectra with $R \sim 20,000$ and $S / N \sim 50 / 1$ per pixel.

\section{HERES}

The Hamburg/ESO R-process Enhanced Star survey (HERES; Christlieb et al. 2004; Barklem et al. 2005) is an ESO Large Programme carried out with VLT UT2/UVES. A total of 373 confirmed metal-poor stars, including four comparison stars, have been observed in "snapshot" mode. The spectra cover a wavelength range of 3760-4980 А. Typical exposure times were $\sim 15 \mathrm{~min}$ for a $B=15 \mathrm{mag}$ star, and the targets were observable under unfavourable weather conditions (i.e., bad seeing, thin cirrus, full moon).

The metallicity range covered by the HERES sample is $-3.8<[\mathrm{Fe} / \mathrm{H}]<-1.5 ; 63$ of the stars have $[\mathrm{Fe} / \mathrm{H}]>-2.5$. The higher metallicity stars where included in order to investigate over what $[\mathrm{Fe} / \mathrm{H}]$ range the $\mathrm{r}-\mathrm{I}$ and $\mathrm{r}-\mathrm{II}$ phenomena occur. The magnitude range covered by the full sample is $10.9<B<17.9$, and $83 \%$ of the stars are brighter than $B=16.5 \mathrm{mag}$, so that higher quality spectra of these stars can be obtained with $8 \mathrm{~m}$-class telescopes within reasonable exposure times.

Table 1. New r-II stars discovered in the HERES project. An entry "y" in the column "hq" indicates that high-quality data has been or will soon be obtained for the star.

\begin{tabular}{|c|c|c|c|c|c|c|c|c|c|}
\hline Star & $V$ & $B-V$ & R.A. & Dec & $T_{\text {eff }}$ & $\log g$ & {$[\mathrm{Fe} / \mathrm{H}]$} & {$[\mathrm{Eu} / \mathrm{Fe}]$} & {$[\mathrm{Ba} / \mathrm{Eu}] \mathrm{hq}$} \\
\hline CS 29491-069 & 13.075 & 0.600 & & & 5100 & 2.5 & -2.81 & 1.06 & $-0.71 \quad y$ \\
\hline $\mathrm{CS} 2$ & & & & & 5010 & 2.2 & & & 0.41 \\
\hline 01 & & 0.584 & 043 & 2 & 5300 & 3.1 & 2.72 & & -0.65 \\
\hline 23 & .165 & 0.732 & 04342 & 091650 & 5130 & 2.6 & -3.19 & 1.25 & -0.53 \\
\hline HE $1127-1143$ & 15.885 & 0.8 & 112950 & -120013 & 5220 & 2.6 & -2.73 & 1.08 & -0.45 \\
\hline HE $1219-0312$ & 15.940 & 0.641 & 122134.1 & -032840 & 5140 & 2.4 & -2.81 & 1.41 & -0.91 \\
\hline HE $2224+0143$ & 13.680 & 0.714 & 222723.0 & +015833 & 5200 & 2.7 & -2.58 & 1.05 & -0.46 \\
\hline HE 2327-5642 & 13.881 & 0.709 & 233037.2 & -562614 & 5050 & 2.2 & -2.95 & 1.22 & -0.56 \\
\hline
\end{tabular}

\section{Results}

A total of 253 stars of our sample were analysed with the automated methods of Barklem et al. (2005, see also this volume). In this subsample, we found 35 new r-I and eight new r-II stars. The latter are listed in Table 1. For five of them, high-quality spectra 
either have been obtained, or will be obtained soon. The results for all 253 stars, their coordinates, and photometry, can be found in Barklem et al. (2005).

In one of the stars for which we already have higher-quality VLT/UVES data, CS 29497004, we detected uranium (Hill et al. 2005, in preparation).

As stated above, we define the r-I and r-II phenomena by the $[\mathrm{Eu} / \mathrm{Fe}]$ and $[\mathrm{Ba} / \mathrm{Eu}]$ ratios. However, due to the limited quality of the snapshot spectra, we cannot detect any $\mathrm{Eu}$ lines in stars with moderate $[\mathrm{r} / \mathrm{Fe}]$ and low $[\mathrm{Fe} / \mathrm{H}]$. For such stars, and where the s-process contribution is likely to be small (i.e., $[\mathrm{Fe} / \mathrm{H}]<-2.5$ and $[\mathrm{C} / \mathrm{Fe}]<0.3$ ), we instead use $\mathrm{Ba}$ as an indicator for $[\mathrm{r} / \mathrm{Fe}]$ by comparing the measured $\mathrm{Ba}$ abundance with $[\mathrm{Ba} / \mathrm{Fe}]_{\mathrm{r}-\text { process }}$, the solar r-process fraction of Ba (see Barklem et al. 2005):

$$
\begin{aligned}
{[\mathrm{Ba} / \mathrm{Fe}]_{\mathrm{r}-\text { process }} } & \equiv \log \left(\frac{N_{\mathrm{Ba}}}{N_{\mathrm{Fe}}}\right)_{*}-\log \left(\frac{N_{\mathrm{Ba}, \mathrm{r}-\text { process }}}{N_{\mathrm{Fe}}}\right)_{\odot} \\
& \equiv \log \left(\frac{N_{\mathrm{Ba}}}{N_{\mathrm{Fe}}}\right)_{*}-\left(\log \left(\frac{N_{\mathrm{Ba}}}{N_{\mathrm{Fe}}}\right)_{\odot}-0.72\right) \\
& \equiv[\mathrm{Ba} / \mathrm{Fe}]+0.72
\end{aligned}
$$

We use the solar abundances of Grevesse \& Sauval (1998) and the r-process fractions of Arlandini et al. (1999).

The distribution of $\mathrm{r}-\mathrm{I}$ and $\mathrm{r}-\mathrm{II}$ stars in the $[\mathrm{Ba} / \mathrm{Fe}]_{\mathrm{r}-\text { process }}$ versus $[\mathrm{Fe} / \mathrm{H}]$ diagram is shown in Figure 1. It appears that the $\mathrm{r}-\mathrm{II}$ phenomenon is confined to the range $-3.2<[\mathrm{Fe} / \mathrm{H}]<-2.6$, while moderate r-process enhancements are also seen at higher $[\mathrm{Fe} / \mathrm{H}]$. If we assume that the r-process elements of the r-II stars have their origin in a single nucleosynthesis event, it follows that the ratio of iron to r-process elements produced in this event must be in a certain range, e.g. $\log \epsilon(\mathrm{Fe} / \mathrm{Eu})=5.3-5.9$. This might provide an important constraint for identifying one of the sites of the r-process.

\section{Conclusions}

Previously, four r-II stars were known: CS 22892-052 (Sneden et al. 1996), CS 31082001 (Cayrel et al. 2001), CS 22183-031 (Honda et al. 2004), and HE 1226-1149 (Cohen 2005, private communication). With the eight new r-II stars found in the HERES effort, we have increased the total number of such stars by a factor of three, to now twelve. The HERES results thus demonstrate that it is feasible to efficiently identify r-II and other interesting stars by means of snapshot spectroscopy.

All known r-II stars have metallicities in the range $-3.2<[\mathrm{Fe} / \mathrm{H}]<-2.6$, while $\mathrm{r}-\mathrm{I}$ stars can be found at metallicities as high as $[\mathrm{Fe} / \mathrm{H}]=-1.5$. This needs to be taken into account for future searches for such stars, and it may provide an important constraint for the identification of the site(s) of the r-process(es).

\section{Acknowledgements}

N.C. is grateful for funding through a Henri Chretien International Research Grant administered by the American Astronomical Society, from Deutsche Forschungsgemeinschaft under grant CH 214/3-1, and a Marie Curie Fellowship of the European Community program Improving Human Research Potential and the Socio-Economic Knowledge under contract number HPMF-CT-2002-01437. T.C.B. acknowledges support from grants AST 00-98508, AST 00-98549, AST 04-06784, and PHY 02-16783, Physics Frontiers Centers/JINA: Joint Institute for Nuclear Astrophysics, awarded by the U.S. National 


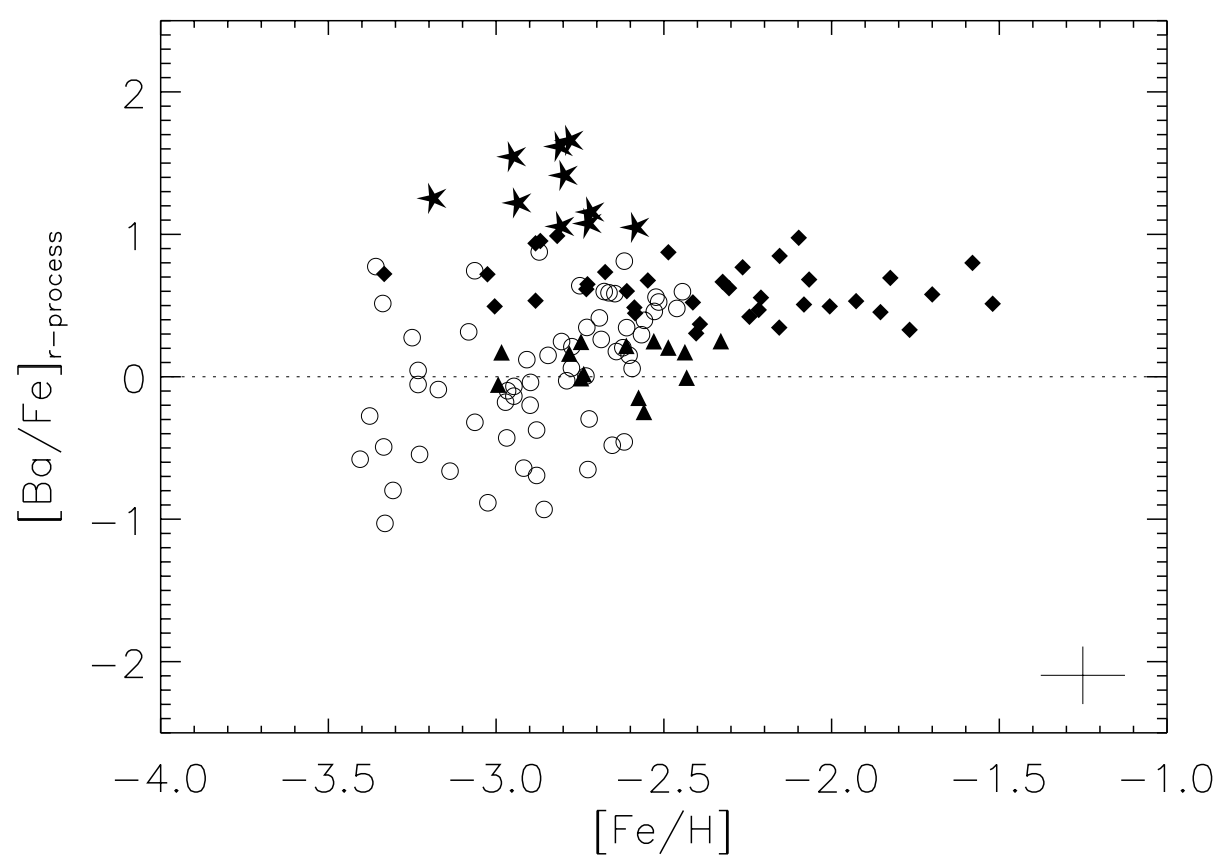

Figure 1. A plot of the enhancement of r-process elements relative to $\mathrm{Fe}$ and the Sun, versus $[\mathrm{Fe} / \mathrm{H}]$. Stars in whose spectra we have detected Eu are shown as filled symbols: r-II stars as stars, r-I stars as diamonds, and stars with $[\mathrm{Ba} / \mathrm{Fe}]<0.0$ and $[\mathrm{Eu} / \mathrm{Fe}]<0.3$ as triangles. For these, we plot $[\mathrm{Eu} / \mathrm{Fe}]$ instead of $[\mathrm{Ba} / \mathrm{Fe}]_{\mathrm{r}-\text { process }}$ (see text for the definition). The r-II stars are those listed in Table 1 and the two comparison stars CS 22892-052 and CS 31082-001. Open circles show stars having $[\mathrm{C} / \mathrm{Fe}]<0.3$ and $[\mathrm{Fe} / \mathrm{H}]<-2.5$, and for which we do not have a significant detection of any Eu lines.

Science Foundation. P.B. acknowledges the support of the Swedish Research Council, and A.J.K. of the Studienstiftung des deutschen Volkes and the Akademie Leopoldina under grant BMBF-LPD 9901/8-87. S.R. thanks the Brazilian Institutions FAPESP and CNPq for partial financial support. K.-L.K. and B.P. are supported by HGF VISTARS VH-VI061. B.N. acknowledges support by the Carlsberg Foundation, the Instrumentcenter for Danish Astrophysics and the Nordic Research Board.

\section{References}

Arlandini, C., Käppeler, F., Wisshak, K., Gallino, R., Lugaro, M., Busso, M., \& Straniero, O.: 1999, Neutron Capture in Low-Mass Asymptotic Giant Branch Stars: Cross Sections and Abundance Signatures, ApJ 525, 886-900.

Barklem, P.S., Christlieb, N., Beers, T.C., Hill, V., Bessell, M.S., Holmberg, J., Marsteller, B., Rossi, S., Zickgraf, F.-J., \& Reimers, D.: 2005, The Hamburg/ESO R-process Enhanced star Survey (HERES). II. Spectroscopic analysis of the survey sample, AEBA, in press.

Beers, T. C., Preston, G. W., \& Shectman, S. A.: 1992, A search for stars of very low metal abundance. II., $A J$ 103, 1987-2034.

Beers, T.C., Preston, G.W., \& Shectman, S.: 1985, A search for stars of very low metal abundance. I., AJ 90, 2089-2102. 
Cayrel, R., Hill, V., Beers, T.C., Barbuy, B., Spite, M., Spite, F., Plez, B., Andersen, J., Bonifacio, P., François, P., Molaro, P., Nordström, B., \& Primas, F.: 2001, Measurement of stellar age from uranium decay, Nature 409, 691-692.

Christlieb, N.: 2003, Finding the Most Metal-poor Stars of the Galactic Halo with the Hamburg/ESO Objective-prism Survey, Rev. Mod. Astron. 16, 191-206. astro-ph/0308016.

Christlieb, N., Beers, T.C., Barklem, P.S., Bessell, M.S., Hill, V., Holmberg, J., Korn, A.J., Marsteller, B., Mashonkina, L., Qian, Y.-Z., Rossi, S., Wasserburg, G.W., Zickgraf, F.-J., Kratz, K.-L., Nordström, B., Pfeiffer, B., Rhee, J., \& Ryan, S.G.: 2004, The Hamburg/ESO R-process Enhanced star Survey (HERES). I. Project description, and discovery of two stars with strong enhancements of neutron-capture elements, A\& $A$ 428, 1043-1049.

Christlieb, N., Bessell, M., Beers, T.C., Gustafsson, B., Korn, A.J., Barklem, P.S., Karlsson, T., Mizuno-Wiedner, M., \& Rossi, S.: 2002, A stellar relic from the early Milky Way, Nature 419, 904-906.

Christlieb, N., Gustafsson, B., Korn, A.J., Barklem, P.S., Beers, T.C., Bessell, M.S., Karlsson, T., \& Mizuno-Wiedner, M.: 2004, HE 0107-5240, a chemically ancient star. I. A detailed abundance analysis, ApJ 603, 708-728. astro-ph/0311173.

Frebel, A., Aoki, W., Christlieb, N., Ando, H., Asplund, M., Barklem, P.S., Beers, T.C., Eriksson, K., Fechner, C., Fujimoto, M., Honda, S., Kajino, T., Minezaki, T., Nomoto, K., Norris, J.E., Ryan, S.G., Takada-Hidai, M., Tsangarides, S., \& Yoshii, Y.: 2005, Nucleosynthetic signatures of the first stars, Nature 434, 871-873.

Grevesse, N., \& Sauval, A.: 1998, Standard Solar Composition, Space Sci. Rev. 85, 161-174.

Honda, S., Aoki, W., Kajino, T., Ando, H., Beers, T.C., Izumiura, H., Sadakane, K., \& TakadaHidai, M.: 2004, Spectroscopic Studies of Extremely Metal-Poor Stars with Subaru/HDS. II. r-Process Elements, Including Thorium, ApJ 607, 474-498.

Sneden, C., McWilliam, A., Preston, G.W., Cowan, J., Burris, D., \& Amorsky, B.: 1996, The ultra-metal-poor, neutron-capture-rich giant star CS 22892-052, ApJ 467, 819-840.

Wisotzki, L., Christlieb, N., Bade, N., Beckmann, V., Köhler, T., Vanelle, C., \& Reimers, D.: 2000, The Hamburg/ESO survey for bright QSOs. III. A large flux-limited sample of QSOs, AESA 358, 77-87. 


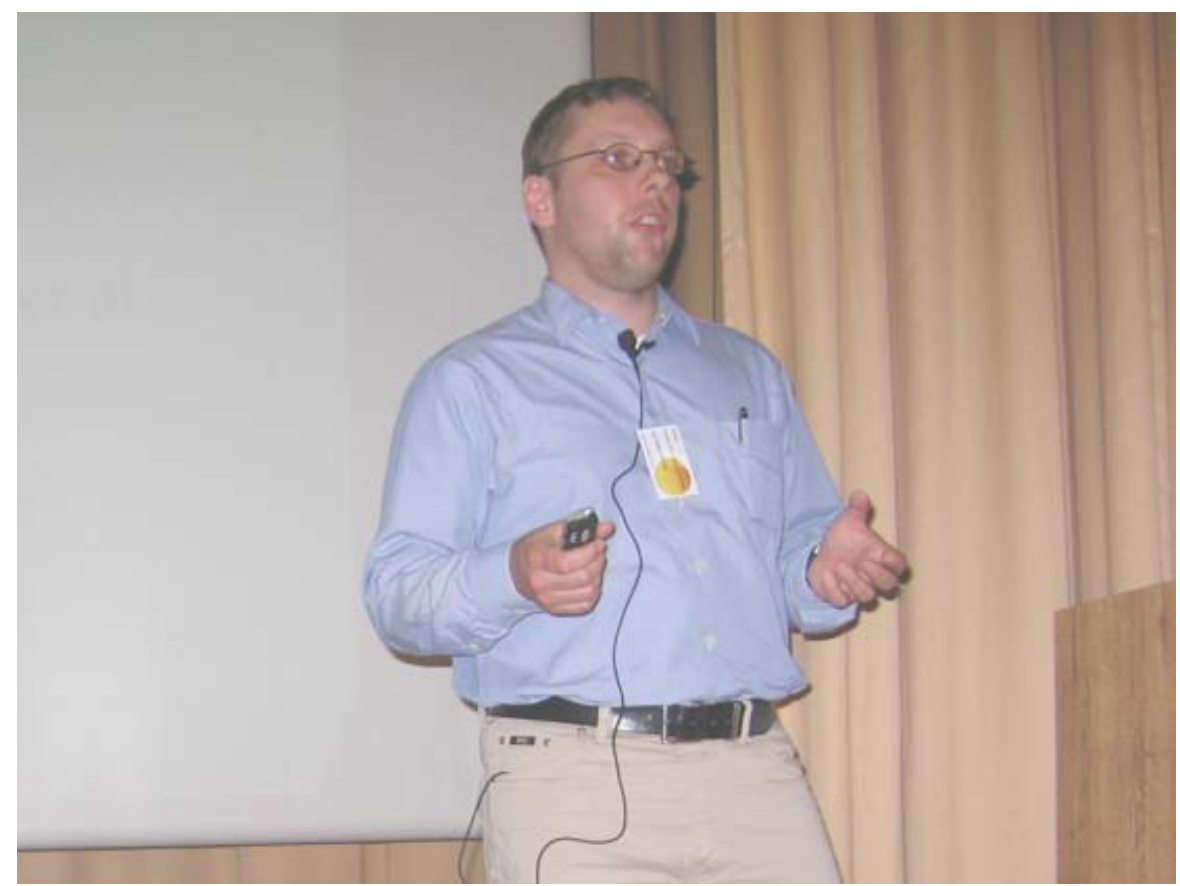

Norbert Christlieb, exposing the results of HERES, a survey for r-process enhancement among metal-poor stars.

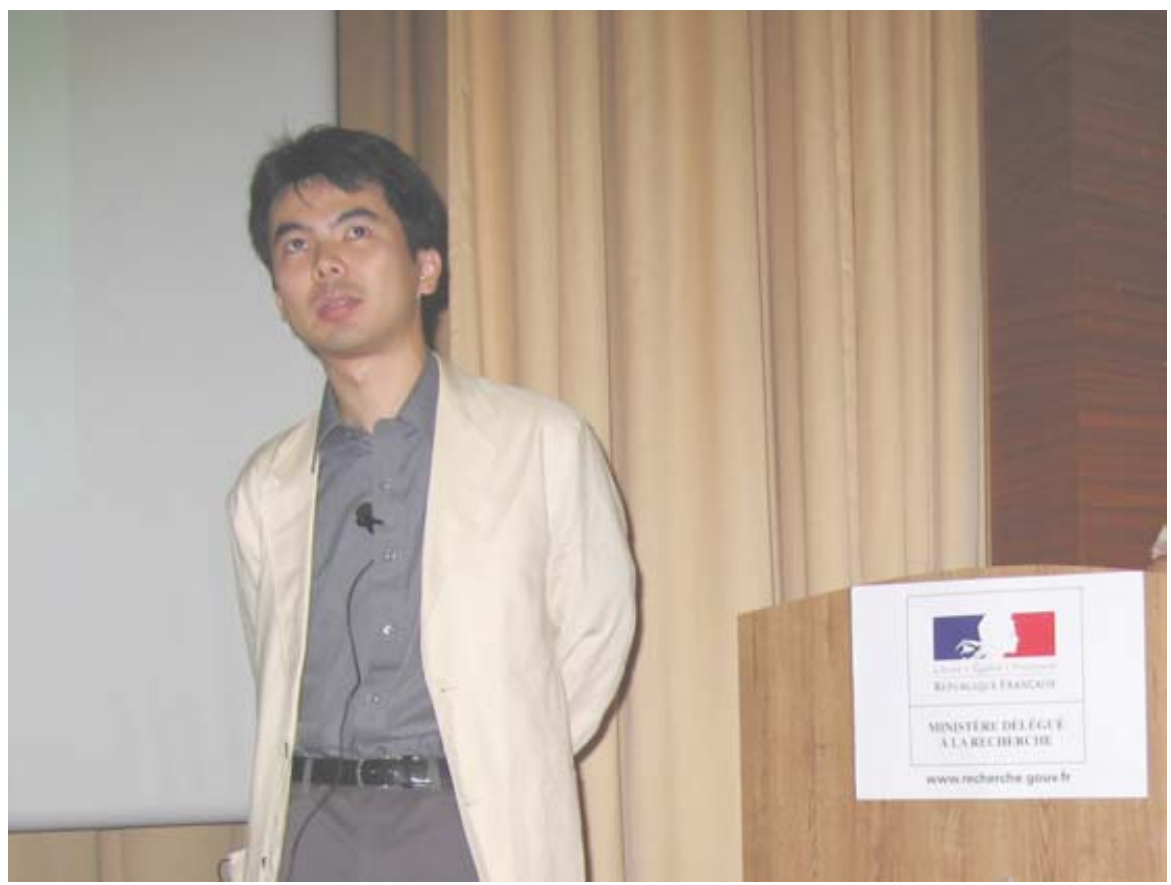

Satoshi Honda presenting the results of heavy elements studies at Subaru. 